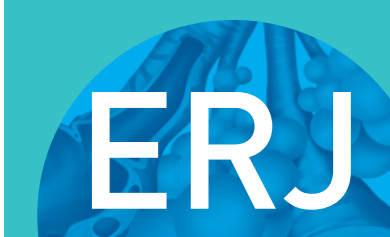

open research

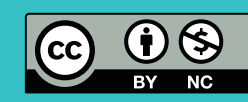

\section{Interstitial lung abnormalities and physical function}

\author{
To the Editor:
}

The term interstitial lung abnormalities (ILA) was coined to define a precursory stage of idiopathic pulmonary fibrosis and encompasses a set of radiological changes seen in interstitial lung diseases (ILD) but are often of lesser magnitude [1]. ILA have been associated with age, many environmental and genetic risk factors and pulmonary symptoms of idiopathic pulmonary fibrosis $[1,2]$. ILA have also been associated with reduced exercise capacity and self-reported health and function [3-5].

Given that ILA are associated with ageing and reduced exercise capacity, the goal of this study was to determine if more diverse markers of physical function were associated with ILA. We proposed five markers of physical function: hand grip strength, knee extension strength, gait speed, the timed up and go test, and thigh muscle mass. These markers are recognised measures of physical function in the elderly [6]. These measures have been associated with mortality [7-9] as well as various markers of disability or poor health outcomes $[10,11]$.

Data from the Icelandic Heart Association's Age, Gene/Environment Susceptibility-Reykjavik Study (AGES-Reykjavik Study) were used. Details of the study design have been previously described [12].

Informed consent was obtained from all participants. The AGES-Reykjavik Study was approved by the National Bioethics Committee in Iceland (VSN: 00-063) and by the National Institute on Aging Intramural institutional Review board [12].

ILA status was determined by jointly evaluating two thoracic computed tomography scans per participant. Each evaluation was carried out by up to three specialised readers; details of this methodology and the definition of ILA have been previously published [13]. Participants were also categorised into one of four subtypes of ILA [1]. The four subtypes were: predominantly centrilobular or peribronchial abnormalities; predominantly subpleural abnormalities; mixed centrilobular and subpleural abnormalities; and radiographic evidence of ILD.

Muscle mass was assessed by quantifying total muscle area in $\mathrm{cm}^{2}$ with computed tomographic imaging, as previously described [9]. The odds ratios provided are per a decrease of $10 \mathrm{~cm}^{2}$. Isometric knee extension strength was measured using an adjustable dynamometer chair as previously described [14]. Hand grip strength of the dominant hand was measured by a computerised dynamometer as previously described [15]. The odds ratios for grip strength and knee extension strength are based on a decrease of 100 Newtons (N). Gait speed was quantified by measuring seconds required to walk a 6-m long course [14]. The tasks included in the timed up and go test were standing up from an arm chair, walking $3 \mathrm{~m}$, walking back to the chair and sitting down again.

Participants with indeterminate ILA status were excluded from statistical analyses $(\mathrm{n}=1726)$, as were participants for which thoracic computed tomography scans were not available $(n=444)$.

Logistic regression modelling was employed for analysis of explanatory variables' association with ILA. Two models were constructed (table 1). One is a univariate model of the association of the explanatory variables with ILA while the other is a multivariable model of the same association in which sex, body mass index (BMI), age, pack-years of smoking and current smoking status were included as covariates.

@ERSpublications

Reductions in objectively measured physical function are associated with interstitial lung abnormalities http://ow.ly/azAW30kMhqv

Cite this article as: Axelsson GT, Putman RK, Miller ER, et al. Interstitial lung abnormalities and physical function. ERJ Open Res 2018; 4: 00057-2018 [https://doi.org/10.1183/23120541.000572018].

The content of this work is not subject to copyright. Design and branding are copyright @ERS 2018. This article is open access and distributed under the terms of the Creative Commons Attribution Non-Commercial Licence 4.0. 
TABLE 1 Associations of functional markers with ILA status and subtypes of ILA

\begin{tabular}{|c|c|c|c|c|c|c|}
\hline & $\begin{array}{l}\text { Subjects } \\
\text { n }(\%)\end{array}$ & $\begin{array}{l}\text { Decreased grip } \\
\text { strength (100 N) }\end{array}$ & $\begin{array}{c}\text { Decreased knee } \\
\text { extension strength } \\
(100 \mathrm{~N})\end{array}$ & $\begin{array}{l}\text { Timed up } \\
\text { and go } \\
\text { test (s) }\end{array}$ & $\begin{array}{l}\text { Gait speed during } \\
\quad 6 \mathrm{~m} \text { walk (s) }\end{array}$ & $\begin{array}{c}\text { Decreased thigh } \\
\text { muscle mass } \\
\left(10 \mathrm{~cm}^{2}\right)\end{array}$ \\
\hline ILA status & 378 & $\begin{array}{c}1.00(0.90-1.11) ; \\
0.99\end{array}$ & $\begin{array}{c}1.04(0.94-1.14) ; \\
0.45\end{array}$ & $\begin{array}{c}1.06(1.03-1.10) \\
0.0001\end{array}$ & $\begin{array}{c}1.09(1.04-1.14) \\
0.0002\end{array}$ & $\begin{array}{c}1.00(0.96-1.05) \\
0.85\end{array}$ \\
\hline \multicolumn{7}{|l|}{ Adjusted model } \\
\hline \multicolumn{7}{|l|}{ ILA subtypes } \\
\hline $\begin{array}{l}\text { Centrilobular } \\
\text { abnormalities }\end{array}$ & $40(10.6)$ & $\begin{array}{c}0.97(0.60-1.58) \\
0.91\end{array}$ & $\begin{array}{c}1.05(0.72-1.54) ; \\
0.79\end{array}$ & $\begin{array}{c}0.96(0.81-1.13) \\
0.64\end{array}$ & $\begin{array}{c}0.94(0.75-1.19) ; \\
0.63\end{array}$ & $\begin{array}{c}0.99(0.77-1.25) \\
0.90\end{array}$ \\
\hline $\begin{array}{l}\text { Subpleural } \\
\text { abnormalities }\end{array}$ & $236(62.4)$ & $\begin{array}{c}1.24(1.02-1.52) \\
0.034\end{array}$ & $\begin{array}{c}1.28(1.08-1.52) ; \\
0.0039\end{array}$ & $\begin{array}{c}1.03 \text { (0.99-1.07); } \\
0.18\end{array}$ & $\begin{array}{c}1.03(0.96-1.10) \\
0.42\end{array}$ & $\begin{array}{c}1.17(1.07-1.28) ; \\
0.0006\end{array}$ \\
\hline $\begin{array}{l}\text { Mixed } \\
\text { abnormalities }\end{array}$ & 85 (22.5) & $\begin{array}{c}1.23(0.89-1.71) ; \\
0.21\end{array}$ & $\begin{array}{c}1.27(0.96-1.68) ; \\
0.097\end{array}$ & $\begin{array}{c}1.04(0.97-1.12) \\
0.24\end{array}$ & $\begin{array}{c}1.12(1.03-1.21) ; \\
0.0071\end{array}$ & $\begin{array}{c}1.09(0.93-1.28) ; \\
0.26\end{array}$ \\
\hline \multicolumn{7}{|c|}{$\begin{array}{l}\text { Data are presented as OR ( } 95 \% \mathrm{CI}) \text {; p-value, unless otherwise stated. Associations were estimated with logistic regression models. Odds ratios } \\
\text { represent the change in interstitial lung abnormalities (ILA) risk per unit change of the variable in question. For example, a } 100 \mathrm{~N} \text { decrease in } \\
\text { grip strength is associated with a change in ILA odds by a factor of } 1.21 \text { in the adjusted model. Unadjusted model: a univariate model of the } \\
\text { association of ILA status to the explanatory variable; adjusted model: a multivariate model adjusted for sex, body mass index (BMI), age, } \\
\text { pack-years and current smoking status as covariates; ILA subtypes: A multivariate model adjusted for sex, BMI, age, pack-years and current } \\
\text { smoking status as covariates. In analyses of each ILA subtype, participants with other subtypes are excluded so the results, given as odds } \\
\text { ratios represent the change in the odds of the specified subtype. ILD: interstitial lung disease. }\end{array}$} \\
\hline
\end{tabular}

Associations with ILA subtypes were also explored (table 1), using the same multivariable logistic models as the general ILA analyses. In modelling of each ILA subtype, participants with other ILA subtypes were excluded. All statistical analyses were done using R.

The results of logistic regression models exploring the associations of functional markers with ILA are displayed in table 1 . As shown in table 1, decreased performance in four out of five markers of physical function was significantly associated with an increased risk of ILA in models adjusted for relevant covariates. These markers were decreased grip strength, decreased knee extension strength, decreased thigh muscle mass and an increase in time required to walk $6 \mathrm{~m}$.

The 378 study participants with ILA were classified into subtypes of ILA as follows: 40 participants (10.6\% of participants with ILA) were determined to have the centrilobular subtype, $236(62.4 \%)$ had subpleural abnormalities, $85(22.5 \%)$ had mixed abnormalities and 17 (4.5\%) had the radiographic interstitial lung disease subtype (table 1).

As shown in table 1, decreased grip strength, knee extension strength and thigh muscle mass were associated with increased risk of the subpleural abnormalities subtype of ILA. Gait speed was inversely associated with the risk of the mixed abnormalities subtype and the radiographic ILD subtype. No markers were significantly associated with the centrilobular subtype of ILA and performance in the timed up and go test did not have significant associations with any of the markers measured.

In this study, we explored the associations of a broader spectrum of physical capabilities with interstitial lung abnormalities than previously reported, demonstrating that decrements in several measures of physical function are associated with increased risk of interstitial lung abnormalities. The associations were found to differ between known subtypes of ILA. These findings support previous studies reporting that ILA are associated with reduced measures of exercise capacity [3, 4] and extend these findings by demonstrating that a broader spectrum of objective physical function measures is associated with ILA.

Although the reasons for the associations presented here are not entirely clear, there are several conceivable explanations for these findings. One plausible possibility is that ILA and declining physical function, at least in some cases, may be driven by similar biological processes, such as age-related processes. A second possibility is that relative reductions in lung capacity [1] and gas exchange [2] could lead to reductions in exercise capacity and strength overall. 
In previous analyses of subtypes of ILA, the subtype associated with the greatest physiological effect was the radiographic ILD subtype while the subpleural abnormalities and mixed abnormalities subtypes were associated with a lesser effect $[1,3]$. Centrilobular predominant ILA is reported to have minimal or no physiological effect $[1,3]$. Our findings are consistent with this prior work, apart from the limited associations of ILA with radiographic ILD. This may be explained by a lack of power to detect these associations, as only 17 individuals had this subtype in the AGES-Reykjavik cohort.

This study has several limitations. The cross-sectional nature of the study makes it impossible to presume causal mechanisms. Although this study demonstrates that those with ILA have reduced measures of physical function by multiple measurements, the biological mechanisms leading to this finding are not known. Additionally, replication of these findings will be important, in part due to the possible existence of unknown confounders or confounders not adjusted for, such as pulmonary function and other comorbidities, and to better define the role of important confounders, such as age, in the findings.

These results support the conclusion that ILA either occurs in people that have worse physical condition than people without ILA, or that ILA results in reduced measures of physical function. Both these suggestions are interesting, especially when viewed in the context of previous reports of people with ILA having an increased risk of mortality [13] and worse subjectively assessed health [5]. Future longitudinal studies exploring the timing of these associations would be of interest.

In conclusion, markers of decreased physical function were found to be associated to interstitial lung abnormalities. These associations differed among subtypes of ILA. The findings suggest that ILA's effect on physical capabilities is greater than previously described and have implications for further research.

Gisli Thor Axelsson ${ }^{1,2,8}$, Rachel K. Putman ${ }^{3,8}$, Ezra R. Miller ${ }^{3}$, Tetsuro Araki ${ }^{4,5}$, Sigurdur Sigurdsson ${ }^{1}$, Elias F. Gudmundsson $\oplus^{1}$, Gudny Eiríksdottír ${ }^{1}$, Kristin Siggeirsdottir ${ }^{1}$, Thor Aspelund $\odot^{1,2}$, Lenore J. Launer ${ }^{6}$, Tamara B. Harris ${ }^{6}$, Hiroto Hatabu ${ }^{4,5}$, Vilmundur Gudnason ${ }^{1,2}$, Gary M. Hunninghake ${ }^{3,5}$ and Gunnar Gudmundsson ${ }^{2,7}$

${ }^{1}$ Icelandic Heart Association, Kopavogur, Iceland. ${ }^{2}$ University of Iceland, Faculty of Medicine, Reykjavik, Iceland. ${ }^{3}$ Pulmonary and Critical Care Division, Brigham and Women's Hospital, Harvard Medical School, Boston, MA, USA. ${ }^{4}$ Dept of Radiology, Brigham and Women's Hospital, Harvard Medical School, Boston, MA, USA. ${ }^{5}$ Center for Pulmonary Functional Imaging, Brigham and Women's Hospital, Harvard Medical School, Boston, MA, USA. ${ }^{6}$ Intramural Research Program, National Institute of Aging, National Institutes of Health, Bethesda, MD, USA. ${ }^{7}$ Dept of Respiratory Medicine, Landspitali University Hospital, Reykjavik, Iceland. ${ }^{8}$ Both authors contributed equally.

Correspondence: Gunnar Gudmundsson, Dept of Respiratory Medicine, Landspitali University Hospital, E7 Fossvogur, IS-108 Reykjavik, Iceland. E-mail: ggudmund@landspitali.is

Received: Feb 132018 | Accepted after revision: July 012018

Author Contributions: G. Gudmundsson, V. Gudnason, H. Hatabu and G.M. Hunninghake designed the study; T. Araki, T. Aspelund, G.T. Axelsson, G. Eiríksdottír, E.F. Gudmundsson, G. Gudmundsson, V. Gudnason, H. Hatabu, G.M. Hunninghake, R.K. Putman, K. Siggeirsdottir and S. Sigurdsson acquired, analysed or interpreted the data; T. Araki, T. Aspelund, G.T. Axelsson, G. Eiríksdottír, E.F. Gudmundsson, G. Gudmundsson, V. Gudnason, T.B. Harris, H. Hatabu, G.M. Hunninghake, L.J. Launer, E.R. Miller, R.K. Putman and S. Sigurdsson critically revised the manuscript for important intellectual content; G.T. Axelsson, G.M. Hunninghake and R.K. Putman performed statistical analysis; and G. Gudmundsson, V. Gudnason and G.M. Hunninghake obtained funding.

Conflict of interest: H. Hatabu reports receiving grants from Toshiba Medical Systems Inc. and Konica-Minolta Inc., and has served on an advisory board for Toshiba Medical System Inc., outside the submitted work. G.M. Hunninghake reports receiving personal fees from Genentech, Boehringer Ingelheim and the Gerson Lehrman Group, outside the submitted work.

Support statement: R.K. Putman is supported by a National Institutes of Health (NIH) grant (T32 HL007633) G. Gudmundsson is supported by a project grant from the Icelandic Research Fund (141513-051) and from the Landspitali Scientific Fund (A-2015-030 and A-2016-023). The AGES (Age, Gene/Environment Susceptibility)-Reykjavik study was supported by a National Institute on Aging (NIA) grant (27120120022C), two NIH contracts (N01-AG-1-2100 and HHSN27120120022C), the NIA Intramural Research Program, Hjartavernd (the Icelandic Heart Association) and the Althingi (the Icelandic Parliament). V. Gudnason is supported by an NIA grant (27120120022C) and an Icelandic Research Fund project grant (141513-051). G.M. Hunninghake is supported by NIH grants (P01 HL114501, R01 HL111024, R01 HL135142 and RO1 HL130974) and a project grant from the Icelandic Research Fund (141513-051). Funding information for this article has been deposited with the Crossref Funder Registry.

\section{References}

1 Washko GR, Hunninghake GM, Fernandez IE, et al. Lung volumes and emphysema in smokers with interstitial lung abnormalities. N Engl J Med 2011; 364: 897-906. 
2 Hunninghake GM, Hatabu $\mathrm{H}$, Okajima $\mathrm{Y}$, et al. MUC5B promoter polymorphism and interstitial lung abnormalities. N Engl J Med 2013; 368: 2192-2200.

3 Doyle TJ, Washko GR, Fernandez IE, et al. Interstitial lung abnormalities and reduced exercise capacity. Am J Respir Crit Care Med 2012; 185: 756-762.

4 Podolanczuk AJ, Oelsner EC, Barr RG, et al. High attenuation areas on chest computed tomography in community-dwelling adults: the MESA study. Eur Respir J 2016; 48: 1442-1452.

5 Axelsson GT, Putman RK, Araki T, et al. Interstitial lung abnormalities and self-reported health and functional status. Thorax 2018; 73: 884-886.

6 Cruz-Jentoft AJ, Baeyens JP, Bauer JM, et al. Sarcopenia: European consensus on definition and diagnosis: Report of the European Working Group on Sarcopenia in Older People. Age Ageing 2010; 39: 412-423.

7 Cooper R, Kuh D, Hardy R, et al. Objectively measured physical capability levels and mortality: systematic review and meta-analysis. BMJ 2010; 341: c4467.

8 Loprinzi PD. Lower extremity muscular strength, sedentary behavior, and mortality. AGE 2016; 38: 32.

9 Reinders I, Murphy RA, Brouwer IA, et al. Muscle quality and myosteatosis: novel associations with mortality risk: the Age, Gene/Environment Susceptibility (AGES)-Reykjavik Study. Am J Epidemiol 2016; 183: 53-60.

10 Cooper R, Kuh D, Cooper C, et al. Objective measures of physical capability and subsequent health: a systematic review. Age Ageing 2011; 40: 14-23.

11 Visser M, Goodpaster BH, Kritchevsky SB, et al. Muscle mass, muscle strength, and muscle fat infiltration as predictors of incident mobility limitations in well-functioning older persons. J Gerontol Ser A 2005; 60: 324-333.

12 Harris TB, Launer LJ, Eiriksdottir G, et al. Age, Gene/Environment Susceptibility - Reykjavik Study: multidisciplinary applied phenomics. Am J Epidemiol 2007; 165: 1076-1087.

13 Putman RK, Hatabu H, Araki T, et al. Association between interstitial lung abnormalities and all-cause mortality. JAMA 2016; 315: 672-681.

14 Reinders I, Murphy RA, Koster A, et al. Muscle quality and muscle fat infiltration in relation to incident mobility disability and gait speed decline: the Age, Gene/Environment Susceptibility-Reykjavik Study. J Gerontol A Biol Sci Med Sci 2015; 70: 1030-1036.

15 Mijnarends DM, Koster A, Schols JM, et al. Physical activity and incidence of sarcopenia: the population-based AGES-Reykjavik Study. Age Ageing 2016; 45: 614-620. 